COMMUNICATIONS IN

ANALYSIS AND GEOMETRY

Volume 7, Number 2, 431-449, 1999

\title{
Degeneration of Kähler-Einstein Metrics on Complete Kähler Manifolds
}

Conan N. Leung and Peng Lu

\section{Introduction.}

According to algebraic geometers, a degeneration of projective varieties is a smooth holomorphic family $\pi: \mathcal{X} \rightarrow \Delta$ with the following property: the fiber $X_{t}=\pi^{-1}(t)$ are smooth except for $t=0$. Assume that the central fiber $X_{0}$ is a reduced divisor with normal crossings. In [T], G. Tian proved the convergence of complete Kähler-Einstein metrics as $t \rightarrow 0$ for two cases: 1) On $X_{t}$ when $X_{t}$ has ample canonical line bundle for $t \neq 0$,2) On $X_{t} \backslash \mathcal{D}$ when $K_{X_{t}}+\mathcal{D} \cap X_{t}$ is ample for $t \neq 0$, where $\mathcal{D}$ is a divisor of $\mathcal{X}$. In case 1 ) the result can be stated as

Theorem (Tian). Let $g_{E, t}$ be Kähler-Einstein metric with

$$
\operatorname{Ric}\left(g_{E, t}\right)=-g_{E, t}
$$

on $X_{t}$. Assume that the central fiber $X_{0}$ is the union of smooth hypersurfaces, say $X_{01}, \cdots, X_{0 m}$, with normal crossings and each line bundle $K_{X_{0 i}}+\sum_{j \neq i} X_{0 j}$ is ample on $X_{0 i}, 1 \leq i \leq m$. Further assume that no three of divisors $X_{0 i}$ have non-empty intersection.

Then $g_{E, t}$ converge to a complete Kähler-Einstein metric on $X_{0} \backslash$ $\operatorname{Sing}\left(X_{0}\right)$ in the sense of Cheeger-Gromov.

In this paper, we prove the same result without assuming that no three divisors have nonempty intersection. The key observation is that Lemma 1.5 in $[\mathrm{T}]$ can be weakened. We will prove our result in a larger setting. Before stating the main theorem of this paper we make several definitions.

Definition 0.1. Let $M$ be a complex manifold of dimension $n$ and $C_{j}$ are smooth hypersurfaces in $M\left(j=1, \cdots, n_{2}\right)$ such that $\sum_{j=1}^{n_{2}} C_{j}$ is a normal crossing divisor. Let $m_{j}$ be natural numbers. Complex V-manifold $M\left(\sum \frac{1}{m_{j}} C_{j}\right)$ is defined in the following way. 
(i) As a topological space, $M\left(\sum \frac{1}{m_{j}} C_{j}\right)$ is $M$,

(ii) For a point $p \in M \backslash \cup C_{j}$, we take a small neighborhood $U$ disjoint from $\cup C_{j}$, and consider $(U, i d)$ as a local uniformization,

(iii) For a point $p$ in some $C_{j}$, without loss of generality, assume $p \in$ $\left(\cap_{1}^{k} C_{j}\right) \backslash\left(\cup_{k+1}^{n_{2}} C_{j}\right)$ for some k. We take a small neighborhood $U$ of $p$ with coordinate system $\left(z^{1}, \cdots, z^{n}\right)$ such that $C_{j}$ is defined by $z^{j}=0$ for $j=1, \cdots, k$, and $C_{j} \cap U=\emptyset$ for $j=k+1, \cdots, n_{2}$. We define the uniformization of $U$ to be $p_{U}: \tilde{U} \rightarrow U$ with

$$
p_{U}\left(w^{1}, \cdots, w^{n}\right)=\left(\left(w^{1}\right)^{m_{1}}, \cdots,\left(w^{k}\right)^{m_{k}}, w^{k+1}, \cdots, w^{n}\right) .
$$

Given a Kähler metric $g$, we will denote its Kähler form by $\omega_{g}$ and its Ricci form by $\operatorname{ric}(g)$.

Definition 0.2. Let $M\left(\sum \frac{1}{m_{j}} C_{j}\right)$ be a complex V-manifold.

(i) A Kähler metric $g$ on $M \backslash \cup C_{j}$ is called a Kähler V-metrics on $M\left(\sum \frac{1}{m_{j}} C_{j}\right)$ if for each $p \in \cup C_{j}, p_{U}^{*} g$ can be extended smoothly to $\tilde{U}$ as a metric.

(ii) A family of Kähler V-metrics $g_{t}$ on $M\left(\sum \frac{1}{m_{j}} C_{j}\right)$ is said to converge in $C^{2}$-topology if $g_{t}$ converge in $C^{2}$-topology on $M \backslash\left(\cup C_{j}\right)$ and $p_{U}^{*} g_{t}$ converge in $C^{2}$-topology on $\tilde{U}$ for each $p \in \cup C_{j}$.

(iii) A Kähler V-metric $g$ on $M\left(\sum \frac{1}{m_{j}} C_{j}\right)$ is called complete if $g$ is a complete metric on $M \backslash \cup U$ with boundary $\partial(\cup U)$ and the extension of $p_{U}^{*} g$ is a complete metric on $\tilde{U}$ with boundary $p_{U}^{-1}(\partial U)$.

(iv) A Kähler V-metric $g$ on $M\left(\sum \frac{1}{m_{j}} C_{j}\right)$ is called Kähler-Einstein if $\operatorname{ric}(g)=-c \cdot \omega_{g}$ on $M \backslash \cup C_{j}$ for some constant $c$.

Definition 0.3. Let $M$ be a smooth projective variety of dimension $\mathrm{n}$ and $D$ be a $\mathbb{Q}$-divisor on $M$.

(i) $D$ is called numerically effective(nef in short) if for any curve $C$ in $M$ the intersection number $D \cdot C$ is non-negative. Such a divisor is called big if $D^{n}>0$. 
(ii) $D$ is called to be ample modulo another divisor $E$ if for every effective reduced curve $C$ on $X$ which is not contained in $E, D \cdot C>0$.

Let $m_{j}$ be a family of natural numbers $\left(1 \leq j \leq n_{2}\right)$. Let $\pi: \mathcal{X} \rightarrow \Delta$ be a degeneration of projective varieties with two divisors $\mathcal{C}$ and $\mathcal{D}$ satisfying the following assumptions.

(1) $X_{t}$ is smooth for $t \neq 0$ and $X_{0}$ is a union of smooth hypersurfaces $X_{0 i}\left(1 \leq i \leq n_{1}\right)$ in $\mathcal{X}$ with normal crossings,

(2) $\mathcal{C}+\mathcal{D}$ is a reduced divisor with normal crossings. Divisor $\mathcal{C}$ consists of smooth components $\mathcal{C}_{1}, \cdots, \mathcal{C}_{n_{2}}$ and divisor $\mathcal{D}$ consists of smooth components $\mathcal{D}_{1}, \cdots, \mathcal{D}_{n_{3}}$. We further assume that the restriction maps $\left.d \pi\right|_{\mathcal{C}_{j}}$ and $\left.d \pi\right|_{\mathcal{D}_{k}}$ are surjective $\left(1 \leq j \leq n_{2}, 1 \leq k \leq n_{3}\right)$,

(3) The divisor $\mathcal{C}+\mathcal{D}$ intersects both central fiber $X_{0}$ and its singular part $\operatorname{Sing}\left(X_{0}\right)$ transversally.

(4) Let $C_{t}=X_{t} \cap \mathcal{C}, C_{j t}=X_{t} \cap \mathcal{C}_{j}$ and $D_{t}=X_{t} \cap \mathcal{D}, D_{k t}=X_{t} \cap \mathcal{D}_{k}$, then each $C_{j t}$ and $D_{k t}$ is smooth. For $t \neq 0$, line bundle

$$
K_{X_{t}}+\sum_{k=1}^{n_{3}} D_{k t}+\sum_{j=1}^{n_{2}} \frac{m_{j}-1}{m_{j}} C_{j t}
$$

is nef, big and ample modulo $D_{t}, t \neq 0$. It follows that there is a unique complete Kähler-Einstein V-metric $g_{E, t}$ on

$$
\left(X_{t} \backslash D_{t}\right)\left(\sum_{j=1}^{n_{2}} \frac{1}{m_{j}} C_{j t}\right)
$$

with $\operatorname{ric}\left(g_{E, t}\right)=-\omega_{g_{E, t}}$ (see [TY], Theorem 2.1 for existence and [Y2], p.474 for completeness).

The following is the main theorem of this paper which provides a suffcient condition on the convergence of this family of metrics $g_{E, t}$ as $t$ goes to 0 .

Theorem 0.1. Let $\pi: \mathcal{X} \rightarrow \Delta$ be the degeneration family with properties (1)-(4) given above. We assume that for each $i, 1 \leq i \leq n_{1}$, line bundle

$$
K_{X_{0 i}}+\sum_{l=1, l \neq i}^{n_{1}} X_{0 l}+\sum_{k=1}^{n_{3}} D_{k 0}+\sum_{j=1}^{n_{2}} \frac{m_{j}-1}{m_{j}} C_{j 0}
$$


is nef, big and ample modulo $\sum_{k=1}^{n_{3}} D_{k 0}$ on $X_{0 i}$. Then the complete KählerEinstein $V$-metric $g_{E, t}$ on $\left(X_{t} \backslash D_{t}\right)\left(\sum_{j=1}^{n_{2}} \frac{1}{m_{j}} C_{j t}\right)$ converges to the unique complete Kähler-Einstein V-metric $g_{E, 0}$ on

$$
\left(X_{0} \backslash\left(\operatorname{Sing}\left(X_{0}\right) \cup D_{0}\right)\right)\left(\sum_{j=1}^{n_{2}} \frac{1}{m_{j}} C_{j 0}\right)
$$

in the sense of Cheeger-Gromov: there are an exhaustion of compact sets $F_{\beta} \Subset X_{0} \backslash\left(\operatorname{Sing}\left(X_{0}\right) \cup D_{0}\right)$ and diffeomorphisms $\phi_{\beta, t}$ from $F_{\beta}$ to into $X_{t}$ satisfying:

i) $X_{t} \backslash\left(D_{t} \cup\left(\cup_{\beta} \phi_{\beta, t}\left(F_{\beta}\right)\right)\right)$ consists of finite union of submanifolds of real codimension 1,

ii) $\phi_{\beta, t}$ maps $C_{0}$ into $C_{t}$ and $\phi_{\beta, t}^{*} g_{E, t}$ is a $V$-metric on $F_{\beta}$,

iii) for each fixed $\beta, V$-metrics $\phi_{\beta, t}^{*} g_{E, t}$ converge to $g_{E, 0}$ on $F_{\beta}$ in $C^{2}$ topology as $t$ goes to 0 .

In section 1 we construct families of Kähler V-metrics on $(\mathcal{X} \backslash(\mathcal{D} \cup$ $\left.\left.\operatorname{Sing}\left(X_{0}\right)\right)\right)\left(\sum_{j=1}^{n_{2}} \frac{1}{m_{j}} C_{j 0}\right)$ with prescribed asymptotic behavior near $X_{0} \backslash$ $\left(\operatorname{Sing}\left(X_{0}\right) \cup D_{0}\right)$. In section 2 we prove Theorem 0.1 using the estimates in [TY] and results from section 1.

The authors thank Gang Tian for helpful discussions.

\section{Construction of family of Kähler V-metrics with asymptotic behavior.}

In this section we adopt the notations used in introduction. For each $i\left(1 \leq i \leq n_{1}\right)$, we choose a neighborhood $U_{i}$ of $X_{0 i}$ in $\mathcal{X}$ such that $\bar{U}_{i_{1}} \cap \bar{U}_{i_{2}}=$ $\emptyset$ when $X_{0 i_{1}} \cap X_{0 i_{2}}=\emptyset$, where $\bar{U}_{i_{1}}$ and $\bar{U}_{i_{2}}$ denote the closure of $U_{i_{1}}$ and $U_{i_{2}}$ respectively. We fix a relative volume $\tilde{V}$ on $\mathcal{X}$. Without loss of generality, we assume $\mathcal{X}=\cup_{i=1}^{n_{1}} U_{i}$. Let $\tilde{V}_{i}$ be the local representation of the relative volume form $\tilde{V}$ on $U_{i}$, in particular, for each $t \in \Delta,\left.\tilde{V}_{i}\right|_{X_{t}}$ is the volume form of $X_{t} \cap U_{i}$. We denote by $U_{i_{1} \cdots i_{l}}$ the intersection $U_{i_{1}} \cap \cdots \cap U_{i_{l}}$ for each tuple $\left(i_{1}, \cdots, i_{l}\right)$. It is a neighborhood of $X_{0 i_{1} \cdots i_{l}}=X_{0 i_{1}} \cap \cdots X_{0 i_{l}}$.

Now we begin to construct a family of Kähler V-metrics with the asymptotic behavior. Let $s_{i}$ be the defining section of line bundle $\left[X_{0 i}\right]$. By Lemma 1.1 and 1.2 in $[\mathrm{T}]$, there are Hermitian metrics \|\|$_{i}$ of line bundles $\left[X_{0 i}\right]$ on $\mathcal{X}$ satisfying $\left(1 \leq i \leq n_{1}\right)$ : 
(1) $\left\|s_{i}\right\|_{i} \equiv 1$ outside $U_{i}$,

(2) $\left\|s_{1}\right\|_{1} \cdots\left\|s_{n_{1}}\right\|_{n_{1}} \equiv|t|$ on $\mathcal{X}$

(3) \|\|$_{i_{1}}^{2} \cdot \tilde{V}_{i_{1}}=\|\|_{i_{2}}^{2} \cdot \tilde{V}_{i_{2}}$ on $U_{i_{1}} \cap U_{i_{2}}, 1 \leq i_{1}, i_{2} \leq n_{1}$.

Without loss of generality, we may assume $\left\|s_{i}\right\|_{i}^{2} \leq 3$ in $\mathcal{X}$. Assume the defining sections of $C_{j}$ and $D_{k}$ in $\mathcal{X}$ are $u_{j}$ and $v_{k}$ respectively. We equip line bundles $\left[C_{j}\right]$ and $\left[D_{k}\right]$ with Hermitian metrics \|\|$_{j, 2}$ and \|\|$_{k, 3}$ respectively. Let $\mu_{1}, \cdots, \mu_{n_{3}}$ be rational numbers in $[0,1]$ and $\varepsilon$ be a small positive number. We will specify them later. Now we define a relative volume $V$ on $\mathcal{X} \backslash\left(\mathcal{C} \cup \mathcal{D} \cup \operatorname{Sing}\left(X_{0}\right)\right)$ as follows. For $t \in \Delta \backslash\{0\}$,

$$
\begin{aligned}
& V_{i t}= \frac{\tilde{V}_{i}}{\prod_{k=1}^{n_{3}}\left[\varepsilon\left\|v_{k}\right\|_{k, 3}^{2 \mu_{k}} \cdot\left(-\log \varepsilon\left\|v_{k}\right\|_{k, 3}^{2}\right)^{2}\right]} \\
& \cdot \frac{1}{\prod_{j=1}^{n_{2}}\left[\varepsilon\left\|u_{j}\right\|_{j, 2}^{\frac{2 m_{j}-2}{m_{j}}} \cdot\left(1-\varepsilon\left\|u_{j}\right\|_{j, 2}^{\frac{2}{m_{j}}}\right)^{2}\right] \cdot \prod_{l=1, l \neq i}^{n_{1}} \varepsilon\left\|s_{l}\right\|_{l}^{2}} \\
& \cdot \prod_{l=1, l \neq i}^{n_{1}}\left[\frac{-\pi}{\log \varepsilon|t|} c s c \frac{\pi \log \varepsilon\left\|s_{l}\right\|_{l}^{2}}{2 \log \varepsilon|t|}\right]^{2} \cdot\left[\frac{-\pi}{\log \varepsilon|t|} \csc \frac{\pi \log \varepsilon \prod_{l=1, l \neq i}^{n_{1}}\left\|s_{l}\right\|_{l}^{2}}{2 \log \varepsilon|t|}\right]^{2} \\
& \quad \text { on } U_{i} \cap\left(X_{t} \backslash\left(C_{t} \cup D_{t}\right)\right)
\end{aligned}
$$

and

$$
\begin{aligned}
& V_{i 0}= \frac{2^{2 n_{1}} \tilde{V}_{i}}{\prod_{k=1}^{n_{3}}\left[\varepsilon\left\|v_{k}\right\|_{k, 3}^{2 \mu_{k}} \cdot\left(-\log \varepsilon\left\|v_{k}\right\|_{k, 3}^{2}\right)^{2}\right]} \\
& \cdot \frac{1}{\prod_{j=1}^{n_{2}}\left[\varepsilon\left\|u_{j}\right\|_{j, 2}^{\frac{2 m_{j}-2}{m_{j}}} \cdot\left(1-\varepsilon\left\|u_{j}\right\|_{j, 2}^{\frac{2}{m_{j}}}\right)^{2}\right] \cdot \prod_{l=1, l \neq i}^{n_{1}} \varepsilon\left\|s_{l}\right\|_{l}^{2}} \\
& \cdot \frac{1}{\prod_{l=1, l \neq i}^{n_{1}}\left(-\log \varepsilon\left\|s_{l}\right\|_{l}^{2}\right)^{2} \cdot\left(-\log \varepsilon \prod_{l=1, l \neq i}^{n_{1}}\left\|s_{l}\right\|_{l}^{2}\right)^{2}} \\
& \quad \text { on } U_{i} \cap\left(X_{0} \backslash\left(C_{0} \cup D_{0} \cup \operatorname{Sing}\left(X_{0}\right)\right)\right) .
\end{aligned}
$$

In order to see that these volume forms $V_{i t}$ can be glued together to give a global volume form $V_{t}$, we simply observe that on

$$
U_{i_{1}} \cap U_{i_{2}} \cap X_{t} \quad\left(1 \leq i_{1}, i_{2} \leq n_{1}\right),
$$




$$
\begin{aligned}
& V_{i_{1} t}=\frac{\tilde{V}_{i_{1}}}{\varepsilon\left\|s_{i_{2}}\right\|_{i_{2}}^{2}} \cdot \frac{1}{\prod_{k=1}^{n_{3}}\left[\varepsilon\left\|v_{k}\right\|_{k, 3}^{2 \mu_{k}} \cdot\left(-\log \varepsilon\left\|v_{k}\right\|_{k, 3}^{2}\right)^{2}\right]} \\
& \cdot \frac{1}{\prod_{j=1}^{n_{2}}\left[\varepsilon\left\|u_{j}\right\|_{j, 2}^{\frac{2 m_{j}-2}{m_{j}}} \cdot\left(1-\varepsilon\left\|u_{j}\right\|_{j, 2}^{\frac{2}{m_{j}}}\right)^{2}\right] \cdot \prod_{l=1, l \neq i_{1}, i_{2}}^{n_{1}} \varepsilon\left\|s_{l}\right\|_{l}^{2}} \\
& \cdot\left[\frac{-\pi}{\log \varepsilon|t|} \csc \frac{\pi \log \varepsilon\left\|s_{i_{2}}\right\|_{i_{2}}^{2}}{2 \log \varepsilon|t|}\right]^{2} \prod_{l=1, l \neq i_{1}, i_{2}}^{n_{1}}\left[\frac{-\pi}{\log \varepsilon|t|} \csc \frac{\pi \log \varepsilon\left\|s_{l}\right\|_{l}^{2}}{2 \log \varepsilon|t|}\right]^{2} \\
& \cdot\left[\frac{-\pi}{\log \varepsilon|t|} \csc \frac{\pi \log \varepsilon \prod_{l=1, l \neq i_{1}}^{n_{1}}\left\|s_{l}\right\|_{l}^{2}}{2 \log \varepsilon|t|}\right]^{2} \\
& =\frac{\tilde{V}_{i_{2}}}{\varepsilon\left\|s_{i_{1}}\right\|_{i_{1}}^{2}} \cdot \frac{1}{\prod_{k=1}^{n_{3}}\left[\varepsilon\left\|v_{k}\right\|_{k, 3}^{2 \mu_{k}} \cdot\left(-\log \varepsilon\left\|v_{k}\right\|_{k, 3}^{2}\right)^{2}\right]} \\
& \frac{1}{\prod_{j=1}^{n_{2}}\left[\varepsilon\left\|u_{j}\right\|_{j, 2}^{\frac{2 m_{j}-2}{m_{j}}} \cdot\left(1-\varepsilon\left\|u_{j}\right\|_{j, 2}^{\frac{2}{m_{j}}}\right)^{2}\right] \cdot \prod_{l=1, l \neq i_{1}, i_{2}}^{n_{1}} \varepsilon\left\|s_{l}\right\|_{l}^{2}} \\
& \cdot\left[\frac{-\pi}{\log \varepsilon|t|} \csc \left(\pi-\frac{\pi \log \left(\varepsilon \prod_{l=1, l \neq i_{2}}^{n_{1}}\left\|s_{l}\right\|_{l}^{2}\right)}{2 \log \varepsilon|t|}\right)\right]^{2} \\
& \cdot \prod_{l=1, l \neq i_{1}, i_{2}}^{n_{1}}\left[\frac{-\pi}{\log \varepsilon|t|} \csc \frac{\pi \log \varepsilon\left\|s_{l}\right\|_{l}^{2}}{2 \log \varepsilon|t|}\right]^{2} \cdot\left[\frac{-\pi}{\log \varepsilon|t|} \csc \left(\pi-\frac{\pi \log \varepsilon\left\|s_{i_{1}}\right\|_{i_{1}}^{2}}{2 \log \varepsilon|t|}\right]^{2}\right. \\
& =V_{i_{2}} \text {. }
\end{aligned}
$$

Define

$$
\begin{array}{ll}
\omega_{t}=\frac{\sqrt{-1}}{2 \pi} \partial \bar{\partial} \log V_{t} & \text { on } X_{t} \backslash\left(C_{t} \cup D_{t}\right) \quad(t \neq 0), \\
\omega_{0}=\frac{\sqrt{-1}}{2 \pi} \partial \bar{\partial} \log V_{0} & \text { on } X_{0} \backslash\left(C_{0} \cup D_{0} \cup \operatorname{Sing}\left(X_{0}\right)\right) .
\end{array}
$$

Simple computations show: for $t \neq 0$, on $U_{i} \cap\left(X_{t} \backslash\left(C_{t} \cup D_{t}\right)\right)$,

$$
\begin{aligned}
\omega_{t}= & -\operatorname{ric}\left(\tilde{V}_{t}\right)+\sum_{k=1}^{n_{3}} \mu_{k} \operatorname{ric}\left(\|\cdot\|_{k, 3}\right)+\sum_{j=1}^{n_{2}} \frac{m_{j}-1}{m_{j}} \operatorname{ric}\left(\|\cdot\|_{j, 2}\right)+\sum_{l=1, l \neq i}^{n_{1}} \operatorname{ric}\left(\|\cdot\|_{l}\right) \\
& +\sum_{k=1}^{n_{3}}\left[\frac{2 \operatorname{ric}\left(\|\cdot\|_{k, 3}\right)}{\log \varepsilon\left\|v_{k}\right\|_{k, 3}^{2}}+\frac{\sqrt{-1}}{\pi} \cdot \frac{\partial \log \left\|v_{k}\right\|_{k, 3}^{2} \wedge \bar{\partial} \log \left\|v_{k}\right\|_{k, 3}^{2}}{\left(-\log \varepsilon\left\|v_{k}\right\|_{k, 3}^{2}\right)^{2}}\right]
\end{aligned}
$$




$$
\begin{aligned}
& +\sum_{j=1}^{n_{2}}\left[\frac{2 \varepsilon \cdot \frac{\sqrt{-1}}{2 \pi} \partial \bar{\partial}\left\|u_{j}\right\|_{j, 2}^{\frac{2}{m_{j}}}}{1-\varepsilon\left\|u_{j}\right\|_{j, 2}^{\frac{2}{m_{j}}}}+\frac{2 \varepsilon^{2} \cdot \frac{\sqrt{-1}}{2 \pi} \partial\left\|u_{j}\right\|_{j, 2}^{\frac{2}{m_{j}}} \wedge \bar{\partial}\left\|u_{j}\right\|_{j, 2}^{\frac{2}{m_{j}}}}{\left(1-\varepsilon\left\|u_{j}\right\|_{j, 2}^{2}\right)^{2}}\right] \\
& +\sum_{l=1, l \neq i}^{n_{1}} \frac{\pi}{\log \varepsilon|t|} \operatorname{ctg} \frac{\pi \log \varepsilon\left\|s_{l}\right\|_{l}^{2}}{2 \log \varepsilon|t|} \cdot \operatorname{ric}\left(\|\cdot\|_{l}\right) \\
& +\sum_{l=1, l \neq i}^{n_{1}}\left(\frac{\pi}{2 \log \varepsilon|t|} \csc \frac{\pi \log \varepsilon\left\|s_{l}\right\|_{l}^{2}}{2 \log \varepsilon|t|}\right)^{2} \cdot \frac{\sqrt{-1}}{\pi} \partial \log \left\|s_{l}\right\|_{l}^{2} \wedge \bar{\partial}\left\|s_{l}\right\|_{l}^{2} \\
& +\frac{\pi}{\log \varepsilon|t|} \operatorname{ctg} \frac{\pi \log \left(\varepsilon \prod_{l=1, l \neq i}^{n_{1}}\left\|s_{l}\right\|_{l}^{2}\right)}{2 \log \varepsilon|t|} \cdot \sum_{l=1, l \neq i}^{n_{1}} \operatorname{ric}\left(\|\cdot\|_{l}\right) \\
& +\left(\frac{\pi}{2 \log \varepsilon|t|} \csc \frac{\pi \log \left(\varepsilon \prod_{l=1, l \neq i}^{n_{1}}\left\|s_{l}\right\|_{l}^{2}\right)}{2 \log \varepsilon|t|}\right)^{2} \\
& . \frac{\sqrt{-1}}{\pi} \partial \log \prod_{l=1, l \neq i}^{n_{1}}\left\|s_{l}\right\|_{l}^{2} \wedge \bar{\partial} \prod_{l=1, l \neq i}^{n_{1}}\left\|s_{l}\right\|_{l}^{2},
\end{aligned}
$$

where $\operatorname{ric}\left(\tilde{V}_{t}\right), \operatorname{ric}\left(\|\cdot\|_{i}\right), \operatorname{ric}\left(\|\cdot\|_{j, 2}\right)$, and $\operatorname{ric}\left(\|\cdot\|_{k, 3}\right)$ denote the curvature tensor of the volume forms $\tilde{V}_{t}=\left.\tilde{V}\right|_{X_{t}}$ and the Hermitian metrics $\|\cdot\|_{i},\|\cdot\|_{j, 2}$, and $\|\cdot\|_{k, 3}$ respectively. Also, on $X_{0 i} \backslash\left(C_{0} \cup D_{0} \cup \operatorname{Sing}\left(X_{0}\right)\right)$,

$$
\begin{aligned}
\omega_{0}= & -\operatorname{ric}\left(\tilde{V}_{0}\right)+\sum_{k=1}^{n_{3}} \mu_{k} \operatorname{ric}\left(\|\cdot\|_{k, 3}\right)+\sum_{j=1}^{n_{2}} \frac{m_{j}-1}{m_{j}} \operatorname{ric}\left(\|\cdot\|_{j, 2}\right)+\sum_{l=1, l \neq i}^{n_{1}} \operatorname{ric}\left(\|\cdot\|_{l}\right) \\
& +\sum_{k=1}^{n_{3}}\left[\frac{2 \operatorname{ric}\left(\|\cdot\|_{k, 3}\right)}{\log \left(\varepsilon\left\|v_{k}\right\|_{k, 3}^{2}\right)}+\frac{\sqrt{-1}}{\pi} \cdot \frac{\partial \log \left\|v_{k}\right\|_{k, 3}^{2} \wedge \bar{\partial} \log \left\|v_{k}\right\|_{k, 3}^{2}}{\left(-\log \varepsilon\left\|v_{k}\right\|_{k, 3}^{2}\right)^{2}}\right] \\
& +\sum_{j=1}^{n_{2}}\left[\frac{2 \varepsilon \cdot \frac{\sqrt{-1}}{2 \pi} \partial \bar{\partial}\left\|u_{j}\right\|_{j, 2}^{\frac{2}{m_{j}}}}{1-\varepsilon\left\|u_{j}\right\|_{j, 2}^{\frac{2}{m_{j}}}}+\frac{2 \varepsilon^{2} \cdot \frac{\sqrt{-1}}{2 \pi} \partial\left\|u_{j}\right\|_{j, 2}^{\frac{2}{m_{j}}} \wedge \bar{\partial}\left\|u_{j}\right\|_{j, 2}^{\frac{2}{m_{j}}}}{\left(1-\varepsilon\left\|u_{j}\right\|_{j, 2}^{2}\right)^{2}}\right] \\
& +\sum_{l=1, l \neq i}^{n_{1}}\left[\frac{2 \cdot \operatorname{ric}\left(\|\cdot\|_{l}\right)}{\log \varepsilon\left\|s_{l}\right\|_{l}^{2}}+\frac{\frac{\sqrt{-1}}{\pi} \cdot \partial \log \left\|s_{l}\right\|_{l}^{2} \wedge \bar{\partial} \log \left\|s_{l}\right\|_{l}^{2}}{\left(\log \varepsilon\left\|s_{l}\right\|_{l}^{2}\right)^{2}}\right] \\
& +\frac{2 \cdot \sum_{l=1, l \neq i}^{n_{1}} \operatorname{ric}\left(\|\cdot\|_{l}\right)}{\log \varepsilon \prod_{l=1, l \neq i}^{n_{1}}\left\|s_{l}\right\|_{l}^{2}} \\
& +\frac{\frac{\sqrt{-1}}{\pi} \partial \log \prod_{l=1, l \neq i}^{n_{1}}\left\|s_{l}\right\|_{l}^{2} \wedge \bar{\partial} \log \prod_{l=1, l \neq i}^{n_{1}}\left\|s_{l}\right\|_{l}^{2}}{\left(\log \varepsilon \prod_{l=1, l \neq i}^{n_{1}}\left\|s_{l}\right\|_{l}^{2}\right)^{2}}
\end{aligned}
$$


Assume that $K_{X_{t}}+\sum_{k=1}^{n_{3}} D_{k t}+\sum_{j=1}^{n_{2}} \frac{m_{j}-1}{m_{j}} C_{j t}$ is nef, big and ample modulo $D_{t}, t \neq 0$. By a result of $\mathrm{Y}$. Kawamata $([\mathrm{K}])$, there are constants $0<\mu_{k}<$ $1\left(1 \leq k \leq n_{3}\right)$ such that for properly chosen volume $\tilde{V}$,

$$
-\operatorname{ric}\left(\tilde{V}_{t}\right)+\sum_{k=1}^{n_{3}} \mu_{k} \operatorname{ric}\left(\|\cdot\|_{k, 3}\right)+\sum_{j=1}^{n_{2}} \frac{m_{j}-1}{m_{j}} \operatorname{ric}\left(\|\cdot\|_{j, 2}\right)
$$

is a Kähler V-metric on $\left(X_{t} \backslash D_{t}\right)\left(\sum \frac{1}{m_{j}} C_{j t}\right)$. Assume that for each $i, 1 \leq$ $i \leq n_{1}$, line bundle

$$
K_{X_{0 i}}+\sum_{k=1}^{n_{3}} D_{k 0}+\sum_{j=1}^{n_{2}} \frac{m_{j}-1}{m_{j}} C_{j 0}+\sum_{l=1, l \neq i}^{n_{1}} X_{0 l}
$$

is nef, big and ample modulo $D_{0}$ on $X_{0 i}$, then

$$
-\operatorname{ric}\left(\tilde{V}_{0}\right)+\sum_{k=1}^{n_{3}} \mu_{k} \operatorname{ric}\left(\|\cdot\|_{k, 3}\right)+\sum_{j=1}^{n_{2}} \frac{m_{j}-1}{m_{j}} \operatorname{ric}\left(\|\cdot\|_{j, 2}\right)+\sum_{l=1, l \neq i}^{n_{1}} \operatorname{ric}\left(\|\cdot\|_{l}\right)
$$

is a Kähler V-metric on $\left(X_{0 i} \backslash\left(D_{0} \cup \operatorname{Sing}\left(X_{0}\right)\right)\right)\left(\sum \frac{1}{m_{j}} C_{j 0}\right)$ for the same reason. The following lemma follows directly from the formulas of $\omega_{t}$ and $\omega_{0}$ above.

Lemma 1.1. Assume that $K_{X_{t}}+\sum_{k=1}^{n_{3}} D_{k t}+\sum_{j=1}^{n_{2}} \frac{m_{j}-1}{m_{j}} C_{j t}$ is nef, big and' ample modulo $D_{t}, t \neq 0$, and assume that for each $i, 1 \leq i \leq n_{1}$, line bundle

$$
K_{X_{0 i}}+\sum_{k=1}^{n_{3}} D_{k 0}+\sum_{j=1}^{n_{2}} \frac{m_{j}-1}{m_{j}} C_{j 0}+\sum_{l=1, l \neq i}^{n_{1}} X_{0 l}
$$

is nef, big and ample modulo $D_{0}$ on $X_{0 i}$.

Then by choosing the volume form $\tilde{V}$ properly and a small $\varepsilon, \omega_{t}$ is the Kähler forms of complete Kähler $V$-metrics $g_{t}$ for $t$ sufficiently small. Moreover, the Kähler $V$-metrics $g_{t}$ converges to $g_{0}$ outside $D_{0} \cup \operatorname{Sing}\left(X_{0}\right)$ in the sense of Cheeger-Gromov: there are an exhaustion of compact subsets $F_{\beta} \Subset X_{0} \backslash\left(D_{0} \cup \operatorname{Sing}\left(X_{0}\right)\right)$ and diffeomorphisms $\phi_{\beta, t}$ from $F_{\beta}$ into $X_{t}$ satisfying:

(1) $X_{t} \backslash \cup_{\beta} \phi_{\beta, t}\left(F_{\beta}\right)$ consists of finite union of submanifolds of real codimension 1,

(2) $\phi_{\beta, t}$ map $F_{\beta} \cap C_{0}$ into $C_{t}$ and $\phi_{\beta, t}^{*} g_{t}$ are $V$-metrics, 
(3) for each fixed $\beta, \phi_{\beta, t}^{*} g_{t}$ converge to $g_{0}$ on $F_{\beta}$ in $C^{2}$-topology on the space of $V$-metrics as $t$ goes to 0 .

Before we state the next lemma, we need a couple of definitions.

Definition 1.1. Let $M\left(\sum \frac{1}{m_{j}} C_{j}\right)$ be the V-manifold in Definition 0.1. Let $B$ be a ball in $\mathbb{C}^{n}$ and $\psi$ is a holomorphic map from $B$ into $M . \psi$ is called a quasi-coordinate map if either $\psi(B) \cap\left(\cup C_{j}\right)=\emptyset$ and $\psi$ is of maximal rank everywhere, or $\psi(B)$ is contained in some uniformizing neighborhood $U$, the lifting $\psi_{U}: B \rightarrow \tilde{U}$ is of maximal rank everywhere, and $\psi$ satisfies $\psi=p_{U} \circ \psi_{U} .(B, \psi)$ is called a local quasi-coordinate of the V-manifold.

Definition 1.2. Let $M\left(\sum \frac{1}{m_{j}} C_{j}\right)$ be a V-manifold and $g$ is a smooth Kähler V-metric on it. We call that $\left(M\left(\sum \frac{1}{m_{j}} C_{j}\right), g\right)$ has bounded geometry of order $k+\beta$, where $k \in \mathbb{N}, \beta \in[0,1)$, if the following conditions hold: There are a system of quasi-coordinates $\left\{\left(B_{\alpha}, \psi_{\alpha}\right)\right\}$ such that:

(i) Every $x \in M$ is the image of the center of some $B_{\alpha}$,

(ii) There are positive numbers $\varepsilon$ and $\delta$ independent of $\alpha$ such that the radius of $B_{\alpha}$ is between $\varepsilon$ and $\delta$.

(iii) There exists constant $C$ such that

$$
\begin{aligned}
& 0<C^{-1}\left(\delta_{i j}\right) \leq\left(g_{\alpha i \bar{j}}\right) \leq C\left(\delta_{i j}\right) \\
& \left|\frac{\partial^{|p|+|q|} g_{\alpha i \bar{j}}}{\partial z_{\alpha}^{p} \partial \bar{z}_{\alpha}^{q}}\right|_{C^{\beta}\left(B_{\alpha}\right)} \leq C
\end{aligned}
$$

for all multi-indexes $p, q$ with $|p|+|q| \leq k$, where $g_{\alpha i \bar{j}}$ is the pullback metric on $B_{\alpha}$ and $|\cdot|_{C^{\beta}\left(B_{\alpha}\right)}$ is the standard Hölder norm.

Lemma 1.2. Same assumptions as Lemma 1.1. Then

(i) the Kähler V-metric $g_{0}$ in Lemma 1.1 has bounded geometry of order $4+\beta$.

(ii) the Kähler V-metrics $g_{t}$ in Lemma 1.1 have uniformly bounded curvature tensors on the uniformization coordinate systems of the $V$ manifold for $t \neq 0$. 
Proof. (i) we prove that $\left(X_{0 i} \backslash\left(D_{0} \cup \operatorname{Sing}\left(X_{0}\right)\right)\right)\left(\sum_{j} \frac{1}{m_{j}} C_{0 j}\right)$ has bounded geometry of order $4+\beta$ for each $i=1, \cdots, n_{1}$. In [TY] it has been proved that $g_{0}$ has bounded geometry on $\left(X_{0 i} \backslash\left(D_{0} \cup U\left(\operatorname{Sing}\left(X_{0}\right)\right)\right)\right)\left(\sum_{j} \frac{1}{m_{j}} C_{0 j}\right)$, where $U\left(\operatorname{Sing}\left(X_{0}\right)\right)$ is a small neighborhood of $\operatorname{Sing}\left(X_{0}\right)$ in $X_{0 i}$. We only need to prove that $g_{0}$ has bounded geometry on $U\left(\operatorname{Sing}\left(X_{0}\right)\right)$.

Pick up a point $x_{0} \in \operatorname{Sing}\left(X_{0}\right) \cap X_{0 i}$, for simplicity, we assume that

$$
x_{0} \in\left(\bigcap_{l=1}^{i_{1}} X_{0 l}\right) \cap X_{0 i} \cap\left(\bigcap_{j=1}^{j_{1}} C_{j 0}\right) \cap\left(\bigcap_{k=1}^{k_{1}} D_{k 0}\right),
$$

$x_{0} \notin X_{0 l}$, for $l>i_{1}, l \neq i, x_{0} \notin C_{j 0}$, for $j>j_{1}$, and $x_{0} \notin D_{k 0}$, for $k>k_{1}$. Take a neighborhood $U_{x_{0}}$ of $x_{0}$ with coordinate system $\left(z^{1}, \cdots, z^{n}\right)$ of $X_{0 i}$ such that $X_{0 l}$ is defined by $z^{l}=0, l=1, \cdots, i_{1}, D_{k 0}$ is defined by $z^{i_{1}+k}=0, k=1, \cdots, k_{1}$, and $C_{j 0}$ is defined by $z^{i_{1}+k_{1}+j}=0, j=1, \cdots, j_{1}$. If we choose $U_{x_{0}}$ small enough, we may assume that $\left\|s_{l}\right\|_{l}=1, l=i_{1}+$ $1, \cdots, n, l \neq i$. On the uniformization $p_{x_{0}}: \tilde{U}_{x_{0}} \rightarrow U_{x_{0}}$ we have coordinate system $\left(w^{1}, \cdots, w^{n}\right)$. Let $\Delta_{\delta}^{*}=\{w \in \mathbb{C}: 0<|w|<\delta\}$ and $\Delta_{\delta}=\{w \in$ $\mathbb{C}:|w|<\delta\}$, then we may identify $\tilde{U}_{x_{0}}$ with $\left(\Delta_{\delta}^{*}\right)^{i_{1}+k_{1}} \times\left(\Delta_{\delta}\right)^{n-i_{1}-k_{1}}$, where $\delta>0$ only depends on $X_{0}, C_{0}$, and $D_{0}$. We will construct quasi-coordinate on $\tilde{U}_{x_{0}}$ such that the pullback of $g_{0}$ has bounded geometry. The following two lemmas are well-known (see e.g. [TY, p. 602]).

Lemma 1.3. The map $\rho_{\theta}: \Delta_{\delta} \rightarrow \Delta_{\delta}^{*}$ with $\rho_{\theta}(w)=\delta \exp \left(\frac{w+\delta}{w-\delta}+\sqrt{-1} \theta\right)$ is a universal covering map of $\Delta_{\delta}^{*}$, where $\theta \in[0,2 \pi)$. The fundamental domain over $\Delta_{\delta}^{*} \backslash\left\{t e^{\sqrt{-1} \theta}: 0<t<\delta\right\}$ is $\left\{w \in \Delta_{\delta}: 0<\delta \operatorname{Im}(w)<\pi|\delta-w|^{2}\right\}$.

Lemma 1.4. For $\eta \in(0,1), \phi_{\eta}: \Delta_{\delta} \rightarrow \Delta_{\delta}$, with $\phi_{\eta}(w)=\delta \frac{w-\delta \eta}{\delta-\eta w}$, is an automorphism mapping $\eta \delta$ to the origin. Furthermore

$$
\begin{aligned}
\left\{w \in \Delta_{\delta}: 0<\delta \operatorname{Im}(w)\right. & \left.<\pi|\delta-w|^{2},|w|^{2}+(-\log r)|\delta-w|^{2}<\delta^{2}\right\} \\
& \subset \bigcup_{0<\eta<1} \phi_{\eta}^{-1}\left(\Delta_{\frac{1}{2} \delta}\right),
\end{aligned}
$$

when $r$ is chosen small enough.

If we shrink $\tilde{U}_{x_{0}}$ to $\left(\Delta_{r \delta}^{*}\right)^{i_{1}+k_{1}} \times\left(\Delta_{r \delta}\right)^{n-i_{1}-k_{1}}$, then it is covered by

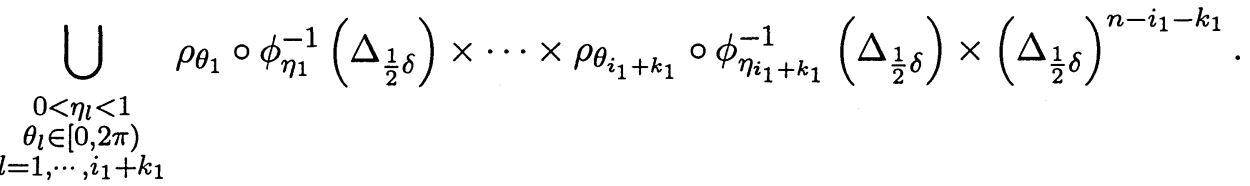


Consider immersion $F: \Delta_{\frac{1}{2} \delta}^{n} \rightarrow \tilde{U}_{x_{0}}$, with

$$
\begin{aligned}
& F\left(v^{1}, \cdots, v^{n}\right) \\
& \quad=\left(\rho_{\theta_{1}} \circ \phi_{\eta_{1}}^{-1}\left(v^{1}\right), \cdots, \rho_{\theta_{i_{1}+k_{1}}} \circ \phi_{\eta_{i_{1}+k_{1}}}^{-1}\left(v^{i_{1}+k_{1}}\right), v^{i_{1}+k_{1}+1}, \cdots, v^{n}\right) .
\end{aligned}
$$

Then by some simple computations, we get

$$
\begin{gathered}
w^{l}=\rho_{\theta_{l}} \circ \phi_{\eta_{l}}^{-1}\left(v^{l}\right)=\delta \exp \left(\frac{\left(1+\eta_{l}\right)\left(v^{l}+\delta\right)}{\left(1-\eta_{l}\right)\left(v^{l}-\delta\right)}+\sqrt{-1} \theta_{l}\right) \\
\frac{\partial}{\partial v^{l}}=-\frac{2 \delta^{2}\left(1+\eta_{l}\right)}{\left(1-\eta_{l}\right)\left(v^{l}-\delta\right)^{2}} \exp \left(\frac{\left(1+\eta_{l}\right)\left(v^{l}+\delta\right)}{\left(1-\eta_{l}\right)\left(v^{l}-\delta\right)}+\sqrt{-1} \theta_{l}\right) \frac{\partial}{\partial w^{l}} \\
\log \left|w^{l}\right|^{2}=2 \log \delta+2 \frac{\left(1+\eta_{l}\right)\left(\left|v^{l}\right|^{2}-\delta^{2}\right)}{\left(1-\eta_{l}\right)\left|v^{l}-\delta\right|^{2}}
\end{gathered}
$$

for $l=1, \cdots, i_{1}+k_{1}$.

On $\tilde{U}_{x_{0}}$, we can write $\omega_{0}=\omega_{0}^{\prime}+\omega_{0}^{\prime \prime}$, where

$$
\begin{aligned}
\omega_{0}^{\prime \prime}= & \sum_{k=1}^{k_{1}} \frac{\frac{\sqrt{-1}}{\pi} \cdot \partial \log \left\|v_{k}\right\|_{k, 3}^{2} \wedge \bar{\partial} \log \left\|v_{k}\right\|_{k, 3}^{2}}{\left(\log \varepsilon\left\|v_{k}\right\|_{k, 3}^{2}\right)^{2}} \\
& +\sum_{l=1}^{i_{1}} \frac{\left.\frac{\sqrt{-1}}{\pi} \cdot \partial \log \left\|s_{l}\right\|_{l}^{2}\right) \wedge \bar{\partial} \log \left\|s_{l}\right\|_{l}^{2}}{\left(\log \varepsilon\left\|s_{l}\right\|_{l}^{2}\right)^{2}} \\
& +\frac{\frac{\sqrt{-1}}{\pi} \cdot \partial \log \prod_{l=1}^{i_{1}}\left\|s_{l}\right\|_{l}^{2} \wedge \bar{\partial} \log \prod_{l=1}^{i_{1}}\left\|s_{l}\right\|_{l}^{2}}{\left(\log \varepsilon \prod_{l=1}^{i_{1}}\left\|s_{l}\right\|_{l}^{2}\right)^{2}} \\
\omega_{0}^{\prime}= & \omega_{0}-\omega_{0}^{\prime \prime}=\frac{\sqrt{-1}}{2 \pi} \sum_{i, j=1}^{n} b_{i \bar{j}} d w^{i} \wedge d \bar{w}^{j} .
\end{aligned}
$$

Then $\omega_{0}^{\prime}$ is a positive $(1,1)$-form on $\tilde{U}_{x_{0}}$. By the choice of $\left(w^{1}, \cdots, w^{n}\right)$, we may assume $\left\|v_{k}\right\|_{k, 3}^{2}=h_{i_{1}+k}\left|w^{i_{1}+k}\right|^{2}$ and $\left\|s_{l}\right\|_{l}=h_{l}\left|w^{l}\right|^{2}$ for some positive functions $h_{i}$ on $\tilde{U}_{x_{0}}, i=1, \cdots, i_{1}+k_{1}$. Substituting (1.1), (1.2) and (1.3) into $F^{*} \omega_{0}$, we obtain

$$
\begin{aligned}
F^{*} \omega_{0}= & \frac{\sqrt{-1}}{2 \pi} \sum_{i, j \geq i_{1}+k_{1}+1} b_{i \bar{j}}\left(F\left(v^{1}, \cdots, v^{n}\right)\right) d v^{i} \wedge d \bar{v}^{j} \\
& +\frac{\sqrt{-1}}{\pi} \sum_{l \leq i_{1}+k_{1}}\left[\frac{1}{\left(\delta^{2}-\left|v^{l}\right|^{2}-\frac{\left(1-\eta_{l}\left|v^{l}-\delta\right|^{2}\right.}{2\left(1+\eta_{l}\right)} \cdot \log \left(\varepsilon \delta^{2} h_{l}\right)\right)^{2}}\right.
\end{aligned}
$$




$$
\begin{gathered}
\cdot\left(\delta^{2} d v^{l}-\frac{\left(1-\eta_{l}\right)\left(v_{l}-\delta\right)^{2}}{2\left(1+\eta_{l}\right)} \cdot \partial \log h_{l}\right) \\
\left.\wedge\left(\delta^{2} d \bar{v}^{l}-\frac{\left(1-\eta_{l}\right)\left(\bar{v}_{l}-\delta\right)^{2}}{2\left(1+\eta_{l}\right)} \cdot \bar{\partial} \log h_{l}\right)\right] \\
+4 \delta^{4} \sum_{i, j \leq i_{1}+k_{1}} b_{i \bar{j}}\left(F\left(v^{1}, \cdots, v^{n}\right)\right) \frac{\left(1+\eta_{i}\right)\left(1+\eta_{j}\right)}{\left(1-\eta_{i}\right)\left(1-\eta_{j}\right)\left(v^{i}-\delta\right)^{2}\left(\bar{v}^{j}-\delta\right)^{2}} \\
\cdot \exp \left(\frac{\left(1+\eta_{i}\right)\left(v^{i}+\delta\right)}{\left(1-\eta_{i}\right)\left(v^{i}-\delta\right)}+\sqrt{-1} \theta_{i}+\frac{\left(1+\eta_{j}\right)\left(\bar{v}^{j}+\delta\right)}{\left(1-\eta_{j}\right)\left(\bar{v}^{j}-\delta\right)}-\sqrt{-1} \theta_{j}\right) \\
\cdot \frac{\sqrt{-1}}{2 \pi} d v^{i} \wedge d \bar{v}^{j} \\
-4 \delta^{2} \operatorname{Re}\left[\begin{array}{l}
\sum_{i \leq i_{1}+k_{1}} b_{i \bar{j}}\left(F\left(v^{1}, \cdots, v^{n}\right) \frac{1+\eta_{i}}{\left(1-\eta_{i}\right)\left(v^{i}-\delta\right)^{2}}\right. \\
j \geq i_{1}+k_{1}+1
\end{array}\right. \\
\cdot \frac{\left.\exp \left(\frac{\left(1+\eta_{i}\right)\left(v^{i}+\delta\right)}{\left(1-\eta_{i}\right)\left(v^{i}-\delta\right)}+\sqrt{-1} \theta_{i}\right) \frac{\sqrt{-1}}{2 \pi} d v^{i} \wedge d \bar{v}^{j}\right]}{\pi} \cdot \frac{1}{\left(\sum_{l \leq i_{1}} \frac{2\left(1+\eta_{l}\right)\left(\mid v^{l} l^{2}-\delta^{2}\right)}{\left(1-\eta_{l}\right)\left|v^{l}-\delta\right|^{2}}+\log \left(\varepsilon \delta^{2 i_{1}} \prod_{l \leq i_{1}} h_{l}\right)\right)^{2}} \\
\cdot \sum_{l \leq i_{1}}\left(\frac{-2 \delta^{2}\left(1+\eta_{l}\right)}{\left(1-\eta_{l}\right)\left(v^{l}-\delta\right)^{2}} d v^{l}+\partial \log h_{l}\right) \\
\wedge \sum_{l \leq i_{1}}\left(\frac{-2 \delta^{2}\left(1+\eta_{l}\right)}{\left(1-\eta_{l}\right)\left(\bar{v}^{l}-\delta\right)^{2}} d \bar{v}^{l}+\bar{\partial} \log h_{l}\right)
\end{gathered}
$$

From the fact that $\lim x \rightarrow \infty x^{p} \exp (-x)=0$ for any real number $p$, it is east to see that in (1.4) the first two terms are equivalent to a Euclidean metric on $\Delta_{\frac{1}{2} \delta}^{n}$, the next two terms are very small since we may choose $\eta_{l}$ close to 1 , the last term is positive and bounded. It is straight forward to check that $F^{*} \omega_{0}$ has bounded geometry.

(ii) We show that $g_{t}$ has uniformly bounded curvature tensor for $t \neq 0$ small. It is known that outside a neighborhood of $\operatorname{Sing}\left(X_{0}\right), g_{t}$ has uniformly bounded geometry ([TY]). It suffices to bound curvature tensor $R m\left(g_{t}\right)$ on some neighborhood of $\operatorname{Sing}\left(X_{0}\right)$. For any $x_{0} \in \operatorname{Sing}\left(X_{0}\right)$, for simplicity, we assume that $x_{0} \in\left(\cap_{l=1}^{i_{1}} X_{0 l}\right) \cap\left(\cap_{j=1}^{j_{1}} C_{j 0}\right) \cap\left(\cap_{k=1}^{k_{1}} D_{k 0}\right), x_{0} \notin X_{0 l}, l>i_{1}$, $x_{0} \notin C_{j 0}, j>j_{1}$, and $x_{0} \notin D_{k 0}, k>k_{1}$. Take a neighborhood of $x_{0}$ with 
coordinate system $\left(z^{1}, \cdots, z^{n+1}\right)$ in $\mathcal{X}$ such that $X_{0 l}$ is defined by

$$
z^{l}=0, l=n+2-i_{1}, \cdots, n+1
$$

$D_{k}$ is defined by

$$
z^{k}=0, k=1, \cdots, k_{1}
$$

and $C_{j}$ is defined by

$$
z^{k_{1}+j}=0, j=1, \cdots, j_{1}
$$

On the uniformization $p_{x_{0}}: \tilde{U}_{x_{0}} \rightarrow U_{x_{0}}$ we have coordinate system $\left(w^{1}, \cdots, w^{n+1}\right)$. We may identify $\tilde{U}_{x_{0}}$ with $\left(\Delta_{\delta}^{*}\right)^{k_{1}} \times\left(\Delta_{\delta}\right)^{n-k_{1}}$, where $\delta>0$ only depends on $\mathcal{X}, \mathcal{C}$, and $\mathcal{D}$. Then

$$
\begin{aligned}
& \tilde{U}_{x_{0}} \cap p_{x_{0}}^{-1}\left(X_{t}\right) \\
& \quad=\left\{\left(w^{1}, \cdots, w^{n+1}\right): w^{n+2-i_{1}} \cdots w^{n+1}=t,\left|w^{l}\right|<\delta, l=1, \cdots, n+1\right\} .
\end{aligned}
$$

Assume $\left\|v_{k}\right\|_{k}=h_{k}\left|w^{k}\right|^{2}$ and $\left\|s_{l}\right\|_{l}=h_{l}\left|w^{l}\right|^{2}$ for $k=1, \cdots, k_{1}, l=n+2-$ $i_{1}, \cdots, n+1$. By the definition of metric $g_{t}$, we have (here we take $i=n+1$ in the definition of $V_{i t}$ )

$$
\begin{gathered}
p_{x_{0}}^{*} \omega_{t}=\frac{\sqrt{-1}}{2 \pi} \partial \bar{\partial} \log \left[\frac{b}{\prod_{k=1}^{k_{1}} \varepsilon\left|w^{k}\right|^{2 \mu_{k}}\left(-\log \left(h_{k}\left|w^{k}\right|^{2}\right)\right)^{2}}\right. \\
\cdot \frac{1}{\prod_{j=1}^{j_{1}} \varepsilon\left|w^{k_{1}+j}\right|^{2} \prod_{l=n+2-i_{1}}^{n} \varepsilon\left|w^{l}\right|^{2}} \\
\cdot \prod_{l=n+2-i_{1}}^{n}\left(\frac{\pi}{\log \varepsilon|t|} c s c \frac{\pi \log \varepsilon h_{l}\left|w^{l}\right|^{2}}{2 \log \varepsilon|t|}\right)^{2} \\
\left.\cdot\left(\frac{\pi}{\log \varepsilon|t|} c s c \frac{\pi \log \left(\varepsilon \prod_{l=n+2-i_{1}}^{n} h_{l}\left|w^{l}\right|^{2}\right)}{2 \log \varepsilon|t|}\right)^{2}\right]
\end{gathered}
$$

where $b$ is a smooth function of

$$
w^{1}, \bar{w}^{1}, \cdots, w^{n}, \bar{w}^{n}, \frac{t}{\prod_{l=n+2-i_{1}}^{n} w^{l}}, \frac{\bar{t}}{\prod_{l=n+2-i_{1}}^{n} \bar{w}^{l}}
$$

with $\frac{\sqrt{-1}}{2 \pi} \partial \bar{\partial} \log b$ positive definite. By exchanging $w^{n+1}$ with one of $w^{n+2-i_{1}}, \cdots, w^{n}$ if necessary, we may assume $\left|w^{l}\right| \geq \sqrt{|t|}, l=n+2-$ $i_{1}, \cdots, n$. Then simple computations show

$$
\omega_{t}=\frac{\sqrt{-1}}{2 \pi} \sum_{\alpha, \beta=1}^{n+1} h_{\alpha \bar{\beta}} d w^{\alpha} \wedge d \bar{w}^{\beta}-\frac{\sqrt{-1}}{2 \pi} \sum_{k=1}^{k_{1}} \partial \bar{\partial} \log \left(\log \varepsilon h_{k}\left|w^{k}\right|^{2}\right)^{2}
$$




$$
\begin{aligned}
& +\frac{\sqrt{-1}}{2 \pi} \sum_{l=n+2-i_{1}}^{n} \partial \bar{\partial} \log \left(\frac{\pi}{\log \varepsilon|t|} \csc \frac{\pi \log \varepsilon h_{l}\left|w^{l}\right|^{2}}{2 \log \varepsilon|t|}\right)^{2} \\
& +\frac{\sqrt{-1}}{2 \pi} \partial \bar{\partial} \log \left(\frac{\pi}{\log \varepsilon|t|} \csc \frac{\pi \log \left(\varepsilon \prod_{l=n+2-i_{1}}^{n} h_{l}\left|w^{l}\right|^{2}\right)}{2 \log \varepsilon|t|}\right)^{2} \\
& =\frac{\sqrt{-1}}{2 \pi} \sum_{\alpha, \beta=1}^{n+1} h_{\alpha \bar{\beta}} d w^{\alpha} \wedge d \bar{w}^{\beta} \\
& +\frac{\sqrt{-1}}{\pi} \sum_{k=1}^{k_{1}} \frac{\left(\frac{d w^{k}}{w^{k}}+\partial \log h_{k}\right) \wedge\left(\frac{d \bar{w}^{k}}{\bar{w}^{k}}+\partial \log h_{k}\right)}{\left(\log \varepsilon h_{k}\left|w^{k}\right|^{2}\right)^{2}} \\
& +\frac{\sqrt{-1}}{\pi} \sum_{k=1}^{k_{1}} \frac{\partial \bar{\partial} \log h_{k}\left|w^{k}\right|^{2}}{\left(-\log \varepsilon h_{k}\left|w^{k}\right|^{2}\right)} \\
& -\frac{\sqrt{-1}}{\pi} \sum_{l=n+2-i_{1}}^{n} \frac{\pi}{2 \log \varepsilon|t|} \cdot c t g \frac{\pi \log \varepsilon h_{l}\left|w^{l}\right|^{2}}{2 \log \varepsilon|t|} \cdot \frac{\partial^{2} \log h_{l}}{\partial w^{\alpha} \partial \bar{w}^{\beta}} d w^{\alpha} \wedge d \bar{w}^{\beta} \\
& +\frac{\sqrt{-1}}{\pi} \sum_{l=n+2-i_{1}}^{n}\left(\frac{\pi}{2 \log \varepsilon|t|} \csc \frac{\pi \log \varepsilon h_{l}\left|w^{l}\right|^{2}}{2 \log \varepsilon|t|}\right)^{2} \\
& \cdot\left(\frac{d w^{l}}{w^{l}}+\partial \log h_{l}\right) \wedge\left(\frac{d \bar{w}^{l}}{\bar{w}^{l}}+\bar{\partial} \log h_{l}\right) \\
& -\frac{\sqrt{-1}}{\pi} \cdot \frac{\pi}{2 \log \varepsilon|t|} \cdot \operatorname{ctg} \frac{\pi \log \left(\varepsilon \prod_{l=n+2-i_{1}}^{n} h_{l}\left|w^{l}\right|^{2}\right)}{2 \log \varepsilon|t|} \\
& \cdot \frac{\partial^{2} \log \prod_{l=n+2-i_{1}}^{n} h_{l}}{\partial w^{\alpha} \partial \bar{w}^{\beta}} d w^{\alpha} \wedge d \bar{w}^{\beta} \\
& +\frac{\sqrt{-1}}{\pi}\left(\frac{\pi}{2 \log \varepsilon|t|} \csc \frac{\pi \log \left(\varepsilon \prod_{l=n+2-i_{1}}^{n} h_{l}\left|w^{l}\right|^{2}\right)}{2 \log \varepsilon|t|}\right)^{2} \\
& \cdot \sum_{l=n+2-i_{1}}^{n}\left(\frac{d w^{l}}{w^{l}}+\partial \log h_{l}\right) \wedge \sum_{l=n+2-i_{1}}^{n}\left(\frac{d \bar{w}^{l}}{\bar{w}^{l}}+\bar{\partial} \log h_{l}\right),
\end{aligned}
$$

where $h_{\alpha \bar{\beta}}$ and $\frac{\partial^{2} \log h_{i}}{\partial w^{\alpha} \partial \bar{w}^{\beta}}$ are smooth functions of

$$
w^{1}, \bar{w}^{1}, \cdots, w^{n}, \bar{w}^{n}, \frac{t}{\prod_{l=n+2-i_{1}}^{n} w^{l}}, \frac{\bar{t}}{\prod_{l=n+2-i_{1}}^{n} \bar{w}^{l}}
$$

and $h_{\alpha \bar{\beta}}$ is positive definite on $\tilde{U}_{x_{0}}$. 
Define

$$
\begin{aligned}
\omega_{t}^{\prime}= & \frac{\sqrt{-1}}{2 \pi} \sum_{\alpha, \beta=1}^{n-1} h_{\alpha \bar{\beta}}\left(w^{1}, \bar{w}^{1}, \cdots, w^{n}, \bar{w}^{n}, 0,0\right) d w^{\alpha} \wedge d \bar{w}^{\beta} \\
& +\frac{\sqrt{-1}}{\pi} \sum_{k=1}^{k_{1}} \frac{d w^{k} \wedge d \bar{w}^{k}}{\left(\left|w^{k}\right| \cdot \log \varepsilon h_{k}\left|w^{k}\right|^{2}\right)^{2}} \\
& +\frac{\sqrt{-1}}{\pi} \sum_{l=n+2-i_{1}}^{n}\left(\frac{\pi}{2 \log \varepsilon|t|} \csc \frac{\pi \log \varepsilon h_{l}\left|w^{l}\right|^{2}}{2 \log \varepsilon|t|}\right)^{2} \frac{d w^{l} \wedge d \bar{w}^{l}}{\left|w^{l}\right|^{2}}
\end{aligned}
$$

and

$$
\begin{aligned}
\omega_{t}^{\prime \prime}= & \omega_{t}^{\prime}+\frac{\sqrt{-1}}{\pi}\left(\frac{\pi}{2 \log \varepsilon|t|} \csc \frac{\pi \log \left(\varepsilon \prod_{l=n+2-i_{1}}^{n} h_{l}\left|w^{l}\right|^{2}\right)}{2 \log \varepsilon|t|}\right)^{2} \\
& \cdot \sum_{l=n+2-i_{1}}^{n} \frac{d w^{l}}{w^{l}} \wedge \sum_{l=n+2-i_{1}}^{n} \frac{d \bar{w}^{l}}{\bar{w}^{l}} .
\end{aligned}
$$

Let $g_{t}^{\prime}$ and $g_{t}^{\prime \prime}$ be the metric corresponding to $\omega_{t}^{\prime}$ and $\omega_{t}^{\prime \prime}$ respectively. Using the fact that $\left|w^{l}\right| \geq \sqrt{|t|}, l=n+2-i_{1}, \cdots, n, g_{t}^{\prime}$ has uniformly bounded curvature tensor. $g_{t}^{\prime \prime}$ is equivalent to $g_{t}^{\prime}$ uniformly in $t$, and their difference is bounded in $C^{3}$-norm defined by $g_{t}^{\prime}$. So $g_{t}^{\prime \prime}$ has uniformly bounded curvature tensor. One can check that $\omega_{t}-\omega_{t}^{\prime \prime}$ are uniformly small in $C^{3}$-topology with respect to the metric $g_{t}^{\prime}$ when we choose $\varepsilon$ small. So $g_{t}$ has uniformly bounded curvature tensor on $\tilde{U}_{x_{0}}$. Lemma 1.2 is proved.

Lemma 1.5. For the metric $g_{t}$ in Lemma 1.1, there is a smooth function $f$ on $\mathcal{X} \backslash\left(\mathcal{C} \cup \mathcal{D} \cup \operatorname{Sing}\left(X_{0}\right)\right)$ compatible with the $V$-manifold structure and bounded from above such that

$$
\begin{aligned}
\operatorname{ric}\left(g_{t}\right)+\omega_{t} & =\frac{\sqrt{-1}}{2 \pi} \partial \bar{\partial} f_{t}, \\
\operatorname{ric}\left(g_{0}\right)+\omega_{0} & =\frac{\sqrt{-1}}{2 \pi} \partial \bar{\partial} f_{0},
\end{aligned}
$$

where $f_{t}=\left.f\right|_{X_{t} \backslash\left(C_{t} \cup D_{t}\right)}$ and $f_{0}=\left.f\right|_{X_{0} \backslash\left(C_{0} \cup D_{0} \cup \operatorname{Sing}\left(X_{0}\right)\right)}$. Furthermore $-\Delta_{g_{t}} f_{t} \leq C$ for some constant $C$ independent of $t$.

Proof. We define $f$ by $(t$ may be 0$)$

$$
f_{t}=-\log \frac{\omega_{t}^{n}}{V_{t}}
$$


It suffices to show that $f_{t}$ and $-\Delta_{g_{t}} f_{t}$ are uniformly bounded from above near $\mathcal{D} \cup \operatorname{Sing}\left(X_{0}\right)$. Here we only show that they are uniformly bounded from above near $\operatorname{Sing}\left(X_{0}\right)$, near $\mathcal{D}$ the proof is similar and easier. First we prove that $f$ is bounded from above near $\operatorname{Sing}\left(X_{0}\right)$. Using the coordinate system $\left(w^{1}, \cdots, w^{n+1}\right)$ in the proof of Lemma 1.2(ii), we have $\omega_{t}^{n}=\left(\omega_{t}^{\prime \prime}\right)^{n}(1+h)$, where $h$ is a function with very small values. So we need to prove that $-\log \frac{\left(\omega_{t}^{\prime \prime}\right)^{n}}{V_{t}}$ is uniformly bounded from above.

Since $\left(\omega_{1}+\omega_{2}\right)^{n} \geq \omega_{1}^{n}$ when both $\omega_{1}$ and $\omega_{2}$ are non-negative (1,1)-forms, observing that last term in (1.6) is non-negative, we have

$$
\begin{aligned}
\frac{\left(\omega_{t}^{\prime \prime}\right)^{n}}{V_{t}} & \geq \frac{\left(\omega_{t}^{\prime}\right)^{n}}{V_{t}} \\
& \geq C \cdot \prod_{k=1}^{k_{1}} \frac{1}{\left|w^{k}\right|^{2\left(1-\mu_{k}\right)}} \cdot\left(\frac{-\pi}{2 \log \varepsilon|t|} \csc \frac{\pi \log \varepsilon \prod_{l=n+2-i_{1}}^{n} h_{l}\left|w^{l}\right|^{2}}{2 \log \varepsilon|t|}\right)^{-2},
\end{aligned}
$$

where $C$ is a constant independent of $t$. Since $u \sin \frac{x}{u} \geq \frac{1}{2} x$ for $0<x \leq u$, using the fact that $\left|w_{l}\right| \geq \sqrt{|t|}$, we have

$$
\left(\frac{-\pi}{2 \log \varepsilon|t|} \csc \frac{\pi \log \varepsilon \prod_{l=n+2-i_{1}}^{n} h_{l}\left|w^{l}\right|^{2}}{2 \log \varepsilon|t|}\right)^{-2} \geq C \cdot\left(\sum_{l=n+2-i_{1}}^{n} \log \varepsilon h_{l}\left|w^{l}\right|^{2}\right)^{2} .
$$

So $f$ is bounded from above by a positive constant independent of $t$.

Next we prove $-\Delta_{g_{t}} f_{t} \leq C$. Using the same coordinate system $\left(w^{1}, \cdots, w^{n+1}\right)$ as above. Then by some computations we find

$$
\begin{aligned}
& f_{t}=-\log \frac{\left(\omega_{t}^{\prime \prime}\right)^{n}\left(1+h_{t}\right)}{V_{t}} \\
& =\log \prod_{k=1}^{k_{1}}\left|w^{k}\right|^{2\left(1-\mu_{k}\right)}-\log \left(\frac{\pi}{2 \log \varepsilon|t|} \csc \frac{\pi \log \varepsilon}{\varepsilon \prod_{l=n+2-i_{1}}^{n} h_{l}\left|w^{l}\right|^{2}}\right)^{-2}+\tilde{h_{t}},
\end{aligned}
$$

where $\tilde{h}$ has uniformly bounded $C^{3}$-norm with respect to metric $g_{t}$. However further computations show that both

$$
-\Delta_{g_{t}} \log \prod_{k=1}^{k_{1}}\left|w^{k}\right|^{2\left(1-\mu_{k}\right)}
$$

and

$$
-\Delta_{g_{t}}\left[-\log \left(\frac{\pi}{2 \log \varepsilon|t|} \csc \frac{\pi \log \varepsilon \prod_{l=n+2-i_{1}}^{n} h_{l}\left|w^{l}\right|^{2}}{2 \log \varepsilon|t|}\right)^{-2}\right]
$$


are bounded from above. So the lemma is proved.

\section{Proof of Theorem 0.1 .}

We adopt the notations of section 1 . Now let $g_{E, t}$ be the Kähler-Einstein V-metrics on $\left(X_{t} \backslash D_{t} \cup \operatorname{Sing}\left(X_{0}\right)\right)\left(\sum \frac{1}{m_{j}} C_{j t}\right)$ for each $t \in \Delta$. Then there are smooth V-functions $\varphi_{t}$ on $\left(X_{t} \backslash D_{t} \cup \operatorname{Sing}\left(X_{0}\right)\right)\left(\sum \frac{1}{m_{j}} C_{j t}\right)$ such that

$$
\omega_{E, t}=\omega_{t}+\frac{\sqrt{-1}}{2 \pi} \partial \bar{\partial} \varphi_{t}
$$

where $\omega_{E, t}$ are the Kähler forms associated with $g_{E, t}$. Furthermore, the following equation follows from $\operatorname{ric}\left(g_{E, t}\right)=-\omega_{E, t}$,

$$
\omega_{E, t}^{n}=e^{f_{t}+\varphi_{t}} \omega_{t}^{n}
$$

where $f_{t}$ is defined in Lemma 1.5 .

Lemma 2.1. There is a uniform constant $C$ independent of $t$ such that $\sup \left|\varphi_{t}+f_{t}\right| \leq C$ on $\left(X_{t} \backslash D_{t} \cup \operatorname{Sing}\left(X_{0}\right)\right)\left(\sum \frac{1}{m_{j}} C_{j t}\right)$.

Proof. It follows from maximal principle since by Lemma 1.2

$$
\left(X_{t} \backslash D_{t} \cup \operatorname{Sing}\left(X_{0}\right)\right)\left(\sum \frac{1}{m_{j}} C_{j t}\right)
$$

has uniformly bounded geometry.(See $[\mathrm{TY}]$ or $[\mathrm{B}]$ )

Lemma 2.2. (i) There are two constants $c$ and $C$ both independent of $t$ and $x$ such that $e^{-c \varphi_{t}(x)}\left(n+\Delta_{g_{t}} \varphi_{t}(x)\right) \leq C$.

(ii) For any compact set $K \subset \mathcal{X} \backslash\left(\mathcal{D} \cup \operatorname{Sing}\left(X_{0}\right)\right)$, there is a uniform constant $C_{K}$ depending on $K$ but independent of $t$ and $x$ such that $n+\Delta_{g_{t}} \varphi_{t} \leq C_{K}$.

Proof. (i) Let $x_{0}$ be the point where $e^{-c \varphi_{t}(x)}\left(n+\Delta_{g_{t}} \varphi_{t}(x)\right)$ attains its maximum. Note that in [Y1] this second-order derivative estimate of $\varphi$ is bounded by a constant depending only on $\operatorname{supf}$ and $\sup \left(-\Delta_{g} f\right)$ for the complex 
Monge-Apmére equation $\left(\omega_{g}+\partial \bar{\partial} \varphi\right)^{n}=e^{f+\varphi} \omega_{g}^{n}$. Using Lemma 1.2 and 1.5, we have the following estimate $n+\Delta_{g_{t}} \varphi_{t}\left(x_{0}\right) \leq C$ by the same computations as the second-order derivative estimate in [Y1] using quasi-coordinate system. On the other hand, from Lemma 2.1 and Lemma 1.5, we have $-\varphi_{t}(x) \leq f_{t}(x)+C \leq C^{\prime}+C$. So $e^{-c \varphi_{t}\left(x_{0}\right)}\left(n+\Delta_{g_{t}} \varphi_{t}\left(x_{0}\right)\right)$ is uniformly bounded.

(ii) From Lemma 2.1, for any compact set $K \subset \mathcal{X} \backslash\left(\mathcal{D} \cup \operatorname{Sing}\left(X_{0}\right)\right)$, we can find $C_{K}$ such that for any $x$ in $K,\left|\varphi_{t}(x)\right| \leq C_{K}$. Now (ii) follows form (i).

Corollary 2.1. For any compact set $K \subset \mathcal{X} \backslash\left(\mathcal{D} \cup \operatorname{Sing}\left(X_{0}\right)\right)$, there is a constant $C_{K}$ depending on $K$ but independent of $t$ such that

$$
\sup _{X_{t} \cap K}\left\{\left|\varphi_{t}\right|,\left|\nabla_{g_{t}}^{k} \varphi_{t}\right|: 1 \leq k \leq 3\right\} \leq C_{K} .
$$

Proof. By working in the quasi-coordinate system, we can show that $\varphi_{t}+f_{t}$ has uniformly bounded $C^{2, \alpha}$-norm on any compact set $K$ (the proof is the same as the proof of Lemma 1.4 in [TY]). In the proof we need to use Lemma 2.1 above. The third derivative estimate of $\varphi_{t}$ follows using Lemma 2.2 and the arguments in [Y1].

Now we conclude the proof of Theorem 0.1. For any sequence $\varphi_{t_{i}}$, by a diagonalizing argument using Corollary 2.1 , we can find a subsequence which converges in the sense of Cheeger-Gromov under $C^{2, \frac{1}{2}}$-topology on $\left(X_{0} \backslash D_{0} \cup \operatorname{Sing}\left(X_{0}\right)\right)\left(\sum \frac{1}{m_{j}} C_{j 0}\right)$. Let $\varphi_{t_{i}}$ be any convergent sequence and $\varphi_{\infty}$ be the limit on $\left(X_{0} \backslash D_{0} \cup \operatorname{Sing}\left(X_{0}\right)\right)\left(\sum \frac{1}{m_{j}} C_{j 0}\right)$, then by Lemma 1.1 $g_{E, t_{i}}$ will converge to a Kähler-Einstein V-metric $\tilde{g}_{E, 0}$ outside $\operatorname{Sing}\left(X_{0}\right)$ in the sense of Cheeger-Gromov. We now prove that V-metric $\tilde{g}_{E, 0}$ is complete. Fix a point $P$ on $\left(X_{0} \backslash D_{0} \cup \operatorname{Sing}\left(X_{0}\right)\right)\left(\sum \frac{1}{m_{j}} C_{j 0}\right)$, let $Q$ be another point close to $\operatorname{Sing}\left(X_{0}\right)$ and $\phi_{\beta, t}$ be defined as in Lemma 1.1. Then the distance between $\phi_{\beta, t_{i}}(P)$ and $\phi_{\beta, t_{i}}(Q)$ defined by metric $g_{t_{i}}$ can be chosen arbitrary large if $Q$ is close enough to $\operatorname{Sing}\left(X_{0}\right)$ and $i$ is large enough. This is also true for the distance between $\phi_{\beta, t_{i}}(P)$ and $\phi_{\beta, t_{i}}(Q)$ defined by metric $g_{E, t_{i}}$. On the other hand if we choose $i$ large enough, this distance approaches the distance between $P$ and $Q$ defined by metric $\tilde{g}_{E, 0}$. So $\tilde{g}_{E, 0}$ is complete $([\mathrm{Y} 2]$, p.474). However, the complete Kähler-Einstein Vmetric on $\left(X_{0} \backslash D_{0} \cup \operatorname{Sing}\left(X_{0}\right)\right)\left(\sum \frac{1}{m_{j}} C_{j 0}\right)$ is unique([TY]), $\tilde{g}_{E, 0}=g_{E, 0}$. 
This shows that $\varphi_{t}$ converges to the unique smooth V-function $\varphi_{0}$ on $\left(X_{0} \backslash D_{0} \cup \operatorname{Sing}\left(X_{0}\right)\right)\left(\sum \frac{1}{m_{j}} C_{j 0}\right)$ in the sense of Cheeger-Gromov, so $g_{E, t}$ converge to $g_{E, 0}$ in the sense of Cheeger-Gromov.

Remark. In [T], Tian proved that for degeneration $\pi: \mathcal{X} \rightarrow \Delta$, the Peterson-Weil metrics bounded from above by $\frac{C \cdot|d z|^{2}}{|z|^{2}\left(-\log |z|^{2}\right)^{3}}$ on the punctured disc $\Delta \backslash\{0\}$ for some constant $C$. However we can not prove it for the degeneration family in Theorem 0.1 because we do not have $g_{E, t} \leq C \cdot g_{t}$ for some $C$ independent of $t$.

\section{References.}

[B] S. Bando, Einstein Kähler Metrics of Negative Ricci Curvature on Open Kähler manifolds, in Advanced Studies in Pure Mathematics, 18-II, Academic Press Inc. 1990, 105-136.

[K] Y. Kawamata, Pluricanonical Systems on Minimal Algebraic Varieties, Inv. Math. 79 (1985), 567-588.

[T] G. Tian, Degeneration of Kähler-Einstein Manifolds, I, Proc. Symp. in Pure Math. 54 (1993), 595-609.

[TY] G. Tian and S.T. Yau, Existence of Kähler-Einstein metrics on complete Kähler manifolds and their applications to algebraic geometry, in Mathematical Aspects of String Theory, World Scientific 1987, 574-628.

[Y1] S.T. Yau, On The Ricci Curvature of a Compact Kähler Manifold and the Complex Monge-Ampére Equation, I*, Comm. Pure Appl. Math. 31 (1978), 339-441.

[Y2] S.T. Yau, A splitting theorem and an algebraic geometric characterization of locally Hermitian symmetric spaces, Comm. Analy. and Geom. 1 (1993), 473-486.

UNIVERSITY OF MinNesota

MiNNEAPOLIS, MN 55455 